\title{
Research of Equidistance Cylindrical Projection Applied in Polar Nautical Chart
}

\author{
ZHANG Zhiheng $^{1,2, a^{*}, \text { PENG Rencan }}{ }^{1,2, b}$, DONG Jian ${ }^{1,2, \mathrm{c}}$ and CHU Yonglei ${ }^{1,2, \mathrm{~d}}$ \\ ${ }^{I}$ Department of Hydrography and Cartography, Dalian Naval Academy ,Dalian, China \\ ${ }^{2}$ PLA key laboratory, Dalian, China \\ azzhd1915@163.com, ${ }^{b}$ pengrencan63@163.com, ${ }^{\text {'navydj@163.com, }{ }^{d} \text { 813465767@qq.com }}$
}

\begin{abstract}
Keywords: Equidistance Cylindrical Projection; Polar Chart; Rhumb Line; Measurement on Nautical Chart
\end{abstract}

Abstract: Aims at the problem that the current polar projections are not completely suitable for polar nautical chart plotting and using, and combines with the existing chart plotting and using methods, the paper proposes the idea of choosing Equidistance Cylindrical Projection as the projection of Polar Chart, analyzes the projection applicability and the shortage of its application aspects. For rhumb line plotted and azimuth, distance measurement are the basic function in chart using, therefore, the paper particularly studies these problems and puts forward corresponding solutions. Meanwhile, some example calculations have been done to validate the corresponding solutions, which lays foundations for the projection application in polar chart.

\section{Introduction}

As the basic mathematical foundation of nautical charting, chart projection selection plays an important role in chart plotting[1]. Nowadays, polar chart published by abroad countries mostly adopts two kinds of chart projection, namely Mercator Projection and Gnomonic Projection, generally Mercator Projection is adopted when the sea area range is from latitude 70 degrees to 80 degrees, while, Gnomonic Projection is adopted when the sea area range is from latitude 80 degrees to 90 degrees[2]. Besides, Lambert Conformal Conic Projection, Azimuthal Conformal Projection, Azimuthal Equidistance Projection, Transverse Mercator Projection, Oblique Mercator Projection and Gnomonic Projection are sometimes adopted in the polar chart[3]. However, some research findings show that these above projections are not completely suitable for polar chart plotting and using, there are mainly three reasons.

i. The length and area distortion of Mercator chart are so serious in polar sea area that Mercator Projection is unable to use, even by adjusting the benchmark latitude[4], Mercator Projection still cannot cover the sea area of latitude from 80 degrees to 90 degrees.

ii. Some chart projections such as Conic Projection, Azimuthal Projection, Transverse Mercator Projection, Oblique Mercator Projection, Gnomonic Projection and etc., the shape of these projection meridian is described in straight line while the parallel is described in arc line, which is not convenient for chart plotting and using.

iii. For the complicated calculation and distortion factors of some chart projection, such as Oblique Mercator Projection and Gnomonic Projection, this is not suitable for char plotting[1].

Therefore, the paper puts forward the idea that choosing Equidistance Cylindrical Projection as the projection of polar chart, analyzes the applicability of the projection in polar chart plotting and using, and solves the key technical problems of Equidistance Cylindrical Projection in polar chart projection, namely, rhumb line plotted and azimuth, distance measurement, which lays foundations for the projection application in polar chart. 


\title{
The applicability of Equidistance Cylindrical Projection in polar chart plotting and using
}

\author{
Equidistance Cylindrical Projection
}

Formula (1) is the equation of Equidistance Cylindrical Projection[5].

$$
\left\{\begin{array}{lr}
x=S_{m} & y=r_{0} L \\
m=1 & n=r_{0} / r \\
P=n & \sin \frac{\omega}{2}=\left|\frac{n-1}{n+1}\right|
\end{array}\right.
$$

In the formula, $S_{m}$ represents the meridian arc length from equator to latitude $B . r_{0}$ represents the benchmark parallel radius, $r$ represents the parallel radius of latitude $B . m$ represents the distortion ratio of meridian length, $n$ represents the distortion ratio of parallel length, $P$ represents the distortion ratio of area, $\omega$ represents the largest distortion ratio of angle. When refers to tangent Equidistance Cylindrical Projection, $r_{0}$ should be substituted for the long earth axis $a$.

Equidistance Cylindrical Projection is a kind of cylindrical projection, the cylinder surface of the projection parallels to the earth axis, meridian and parallel are projected to the cylinder surface under the condition of the meridian length keeping unchanged, cylinder surface is then cut off along a meridian and outspreaded. The meridian interval of the projection is equal to the field distance, the meridian and parallel of the projection plane are two straight lines which are mutual perpendicular and equal interval distance. When the earth is considered as a sphere, the meridian and parallel form a square grid, therefore, the projection is also called Square Projection[5]. As for the distortion property, this projection belongs to arbitrary projection, both angle and area exist distortion, length distortion exists in any position and direction except for the direction of meridian and benchmark latitude.

The applicability of Equidistance Cylindrical Projection in polar chart plotting

In consideration of existing chart projection selection, cylindrical projection is mainly used in chart plotting. The meridian and parallel of these projections are straight and perpendicular, which is convenient to subsequent chart plotting and using. Formula (2) is the general equation of cylindrical projection[5].

$\left\{\begin{array}{c}x=f(B) \\ y=C L\end{array}\right.$

In the formula, $C$ represents constant, $x$ and $y$ represent coordinates on the chart, $B$ and $L$ represent latitude and longitude coordinates. Parameter $x$ and $y$ are irrespective, the projection formula is simple and convenient to chart plotting.

With a view to projection distortion, cylindrical projection can be divided into Conformal Cylindrical Projection, Equal area Cylindrical Projection and Any Cylindrical Projection (mainly Equidistance Cylindrical Projection) when according to the character of projection distortion. Table 1, Table 2 and Table 3 represent the distortion tables of the three projections. The following conclusions can be obtained from the three tables.

i. Conformal Cylindrical Projection increases area distortion to keep the angle unchanged.

ii. Equal area Cylindrical Projection increases angle distortion to keep the area unchanged.

iii. On Equidistance Cylindrical Projection plane, angle, area and length distortion approximately equal to each other, namely, the distortion character of Equidistance Cylindrical Projection is between Conformal Cylindrical Projection and Equal area Cylindrical Projection. 
Table 1. The distortion table of Conformal Cylindrical Projection

\begin{tabular}{rcccc}
\hline$\varphi$ & $m$ & $n$ & $P$ & $\omega$ \\
\hline $0^{\circ}$ & 1.000 & 1.000 & 1.000 & 0 \\
$10^{\circ}$ & 1.015 & 1.015 & 1.031 & 0 \\
$20^{\circ}$ & 1.064 & 1.064 & 1.131 & 0 \\
$30^{\circ}$ & 1.154 & 1.154 & 1.331 & 0 \\
$40^{\circ}$ & 1.304 & 1.304 & 1.699 & 0 \\
$50^{\circ}$ & 1.557 & 1.557 & 2.423 & 0 \\
$60^{\circ}$ & 1.995 & 1.995 & 3.980 & 0 \\
$70^{\circ}$ & 2.915 & 2.915 & 8.498 & 0 \\
$80^{\circ}$ & 5.7400 & 5.7400 & 32.947 & 0 \\
$90^{\circ}$ & $\infty$ & $\infty$ & $\infty$ & 0 \\
\hline & & & &
\end{tabular}

Table 2. The distortion table of Equal area Cylindrical Projection

\begin{tabular}{rcccc}
\hline$\varphi$ & $m$ & $n$ & $P$ & $\omega$ \\
\hline $0^{\circ}$ & 1.000 & 1.000 & 1.000 & $0^{\circ} 00^{\prime}$ \\
$10^{\circ}$ & 0.985 & 1.015 & 1.000 & $1^{\circ} 45^{\prime}$ \\
$20^{\circ}$ & 0.940 & 1.064 & 1.000 & $7^{\circ} 07^{\prime}$ \\
$30^{\circ}$ & 0.866 & 1.155 & 1.000 & $16^{\circ} 26^{\prime}$ \\
$40^{\circ}$ & 0.766 & 1.305 & 1.000 & $30^{\circ} 11^{\prime}$ \\
$50^{\circ}$ & 0.643 & 1.556 & 1.000 & $49^{\circ} 04^{\prime}$ \\
$60^{\circ}$ & 0.500 & 2.000 & 1.000 & $73^{\circ} 44^{\prime}$ \\
$70^{\circ}$ & 0.342 & 2.924 & 1.000 & $104^{\circ} 28^{\prime}$ \\
$80^{\circ}$ & 0.174 & 5.579 & 1.000 & $140^{\circ} 36^{\prime}$ \\
$90^{\circ}$ & 0.000 & $\infty$ & 1.000 & $180^{\circ} 00^{\prime}$ \\
\hline
\end{tabular}

Table 3. The distortion table of Equidistance Cylindrical Projection

\begin{tabular}{rcccl}
\hline$\varphi$ & $m$ & $n$ & $P$ & $\omega$ \\
\hline $0^{\circ}$ & 1.000 & 1.000 & 1.000 & $0^{\circ} 00^{\prime}$ \\
$10^{\circ}$ & 1.000 & 1.015 & 1.015 & $0^{\circ} 52^{\prime}$ \\
$20^{\circ}$ & 1.000 & 1.064 & 1.064 & $3^{\circ} 33^{\prime}$ \\
$30^{\circ}$ & 1.000 & 1.155 & 1.155 & $8^{\circ} 14^{\prime}$ \\
$40^{\circ}$ & 1.000 & 1.305 & 1.305 & $15^{\circ} 10^{\prime}$ \\
$50^{\circ}$ & 1.000 & 1.556 & 1.556 & $25^{\circ} 01^{\prime}$ \\
$60^{\circ}$ & 1.000 & 2.000 & 2.000 & $38^{\circ} 57^{\prime}$ \\
$70^{\circ}$ & 1.000 & 2.915 & 2.915 & $58^{\circ} 34^{\prime}$ \\
$80^{\circ}$ & 1.000 & 5.740 & 5.740 & $89^{\circ} 23^{\prime}$ \\
$90^{\circ}$ & 1.000 & $\infty$ & $\infty$ & $180^{\circ} 00^{\prime}$ \\
\hline
\end{tabular}

With a view to the display of projection plane, the parallel interval of Conformal Cylindrical Projection and Equal area Cylindrical Projection is enlarged and reduced significantly in polar sea area, which results the polar sea area cannot be displayed clearly. While, for Equidistance Cylindrical Projection owns equal parallel interval, polar sea area displays better. As shown in Fig.1. 


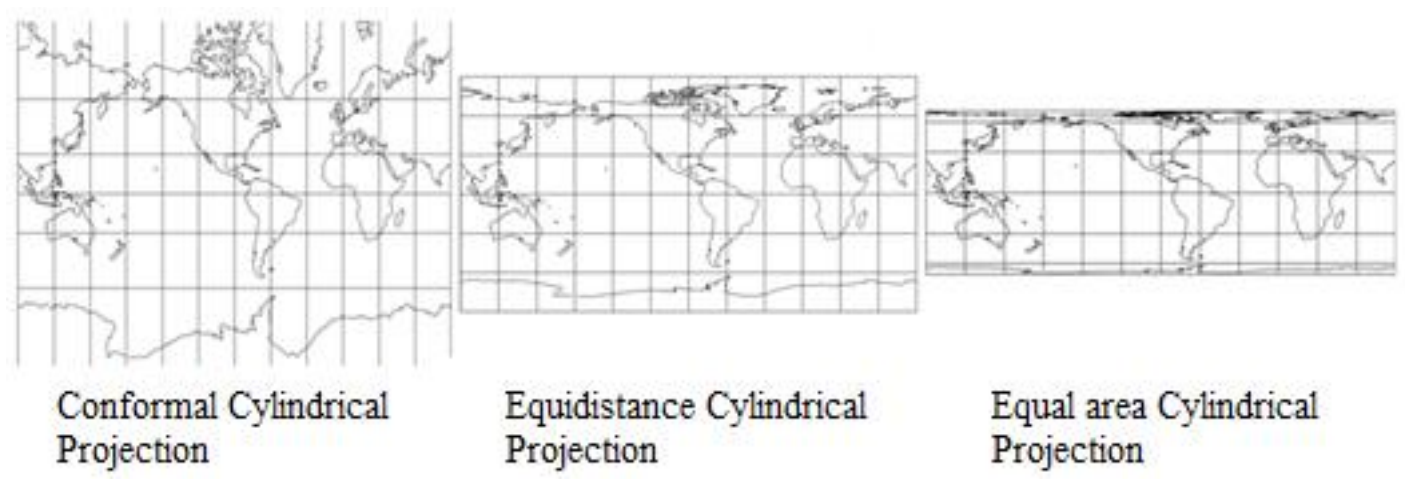

Fig.1 Comparison of three cylindrical projections chart

Based on the above analysis, we can conclude that Equidistance Cylindrical Projection and Mercator Projection are similar in chart plotting, while Equidistance Cylindrical Projection is more suitable for polar chart plotting.

The applicability of Equidistance Cylindrical Projection in polar chart using

As for ship navigation, the usage of chart basically includes positioning on the chart, rhumb line plotted on the chart and Azimuth, distance measurement on the chart, etc.

With a view to positioning on the chart, for meridian and parallel of Equidistance Cylindrical Projection are straight lines and mutual perpendicular, it is just necessary to accurately divide the longitude and latitude according to the formula of Equidistance Cylindrical Projection, therefore, positioning on the chart is very convenient.

With a view to plotting on the chart, rhumb line configuration on Equidistance Cylindrical Projection chart should be analyzed firstly. Formula (3) is the equation of rhumb line on the ellipsoid.

$L_{2}-L_{1}=\tan A\left(q_{2}-q_{1}\right)$

In the formula, isometric latitude $q=\ln U, U=\tan \left(\frac{\pi}{4}+\frac{B}{2}\right)\left(\frac{1-e \sin B}{1+e \sin B}\right)^{e / 2}$.

The formula (4) is the transform equation from meridian arc to isometric latitude[6].

$\left\{\begin{array}{l}\psi=\frac{X}{R} \\ q=Q=\arctan h(\sin \psi)+\xi_{1} \sin \psi+\xi_{3} \sin 3 \psi+\xi_{5} \sin 5 \psi\end{array}\right.$

In the formula, $\psi$ represents equidistance latitude, $X$ represents the meridian arc length calculated from equator, $R$ represents the radius of equidistance earth. In the reference ellipsoid of CGCS 2000, parameter $\xi$ means $\xi_{1}=-1.6742951 \times 10^{-3}, \xi_{3}=-4.680927 \times 10^{-7}$ and $\xi_{5}=-4.32077 \times 10^{-10}$.Substituting Formula(4) in formula (3), and multiplying by the radius of the benchmark parallel $r_{0}=\frac{a \cos B_{0}}{\sqrt{1-e^{2} \sin ^{2} B_{0}}}$ on the both formula side, the equation of rhumb line on Equidistance Cylindrical Projection chart can be calculated as follows.

$Y_{2}-Y_{1}=r_{0} \tan A\left(Q_{2}-Q_{1}\right)$

In the formula, $Q_{1}$ and $Q_{2}$ represent the isometric latitude which are calculated by the ordinate of this projection plane $X_{1}$ and $X_{2}$, or inquired by the Map Projection Calculation Table which can get the corresponding isometric latitude $q$ by the geographic latitude. $Y_{1}$ and $Y_{2}$ represent the abscissa of this projection plane.

When the ship sails along the meridian and parallel, the formula(5) is equal, so the meridian and parallel on the projection plane are two orthogonal rhumb line. In the other directions, it can be 
obtained by the formula (5) that the rhumb line is curve, and it is very difficult for the rhumb line plotted on the chart.

With a view to azimuth, distance measurement on the chart, this projection belongs to arbitrary projection, so angle and length both exist deformation, these cause great difficulty to azimuth, distance measurement on the chart.

Through analyzing the above aspects, The main problems of Equidistance Cylindrical Projection in polar chart using are mainly rhumb line plotted and azimuth, distance measurement on the chart, these problems limit Equidistance Cylindrical Projection in polar chart using. For these key technical problems, the part three and part four will research and solve them and the paper will provide the basis for Equidistance Cylindrical Projection in polar chart plotting and using.

\section{Rhumb line plotted on the chart}

Through the analysis of rhumb line on the chart, the meridian and parallel on the projection plane are two orthogonal rhumb line, plotting on the chart is very convenient. But in the other directions, the rhumb line is curve. In order to plot rhumb line better, the paper uses the method of "drawing curves by straight lines" which is often used in computer graphics, namely, inserting several routes between start and end point, making the precision meet the stipulation of Specifications for Chinese Nautical Charts, which rules that the height between curve and straight line on the chart is not more than $0.1 \mathrm{~mm}$ (the actual distance is $0.0001 C_{0} \mathrm{~m}, C_{0}$ is the chart scale.), so the curve can be replaced by broken line to plot the rhumb line[7].

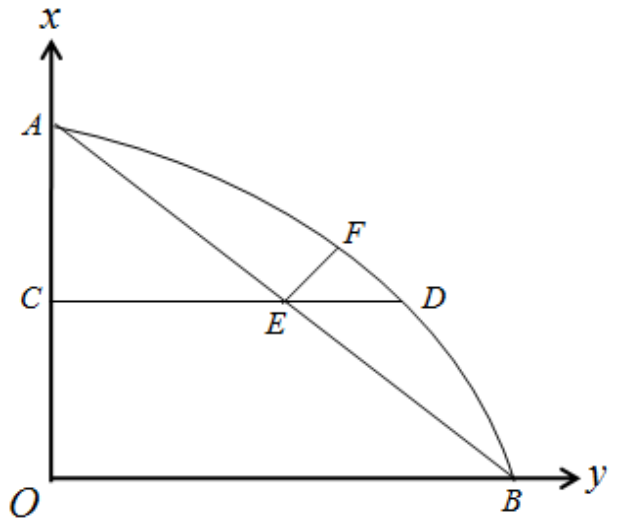

Fig.2 The sketch of rhumb line described

As is shown in Fig.2, arc $A B$ is the rhumb line on Equidistance Cylindrical Projection plane, connecting straight line $A B$, finding the midpoint $C$ of line $A O$, making the line $C D$ parallel to coordinate $y$, straight line $A B$ and $C D$ intersect at point $E$, arc $A B$ and straight line $C D$ intersect at point $D$.Making perpendicular line at point $E$, arc $A B$ and the perpendicular line intersect at point $F$, at last, the Fig.2 will be obtained. In the formula (5), the value of $Q_{1}$ and $Q_{2}$ can be inquired by the Map Projection Calculation Table. The following is the specific description method.

a. Measuring the distance of $O B$ on the $\operatorname{chart}\left(Y_{B}-Y_{A}\right)$, calculating the isometric latitude difference of $A$ and $B$ point $\left(Q_{B}-Q_{A}\right)$, using the formula (5) to calculate $r_{0} \tan A$.

b. Inquiring the isometric latitude of $C$ point, using the formula (5) to calculate the distance of $C D$ on the chart $\left(Y_{D}-Y_{C}\right)$.

c. In the triangle $\triangle A O B$, using $C E=\frac{1}{2} O B$ to get the distance of $C E$ on the $\operatorname{chart}\left(Y_{E}-Y_{C}\right)$.

d. Using the distance of $C D$ and $C E$ to calculate the distance of $E D$. When the distance of $E F$ is less than or equal to $0.0001 C_{0}$, straight line $A B$ can be instead of arc $A B$. Arc $F D$ can be thought paralleling to the straight line $A B$, so $\triangle E F D$ can be thought a right triangle and the distance of $E D$ is less than or equal to $0.0001 C_{0} \sec \angle O A B$. It can be known that when the value of $E D$ is less than or 
equal to $0.0001 C_{0} \sec \angle O A B$, straight line $A B$ can be instead of arc $A B$. If not, connecting $A D$ and $B D$, repeating the above steps on them.

In order to verify the correctness and feasibility of the above method, the paper selects a rhumb line in the Arctic sea area to have an experiment(the Antarctic sea area is same). The sketch of rhumb line in the experiment is shown in Fig.3 and the sketch of interpolation points in rhumb line is shown in Fig.4, geographical coordinates of interpolation points are shown in Table 4.

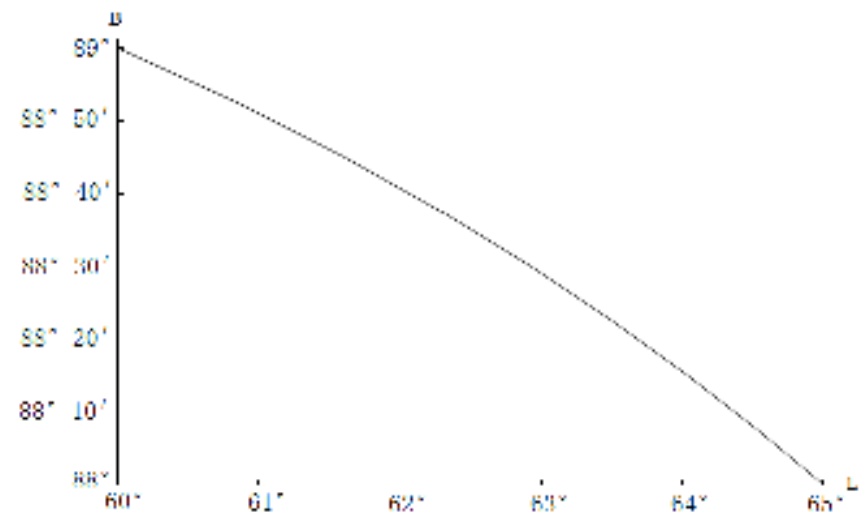

Fig.3 The sketch of rhumb line in the experiment

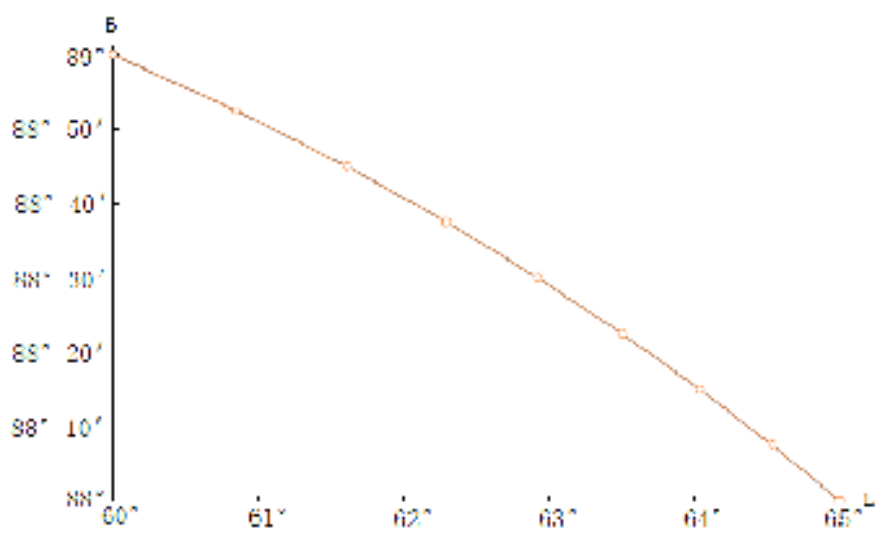

Fig.4 The sketch of interpolation points in rhumb line

Table 4. Geographical coordinates of interpolation points

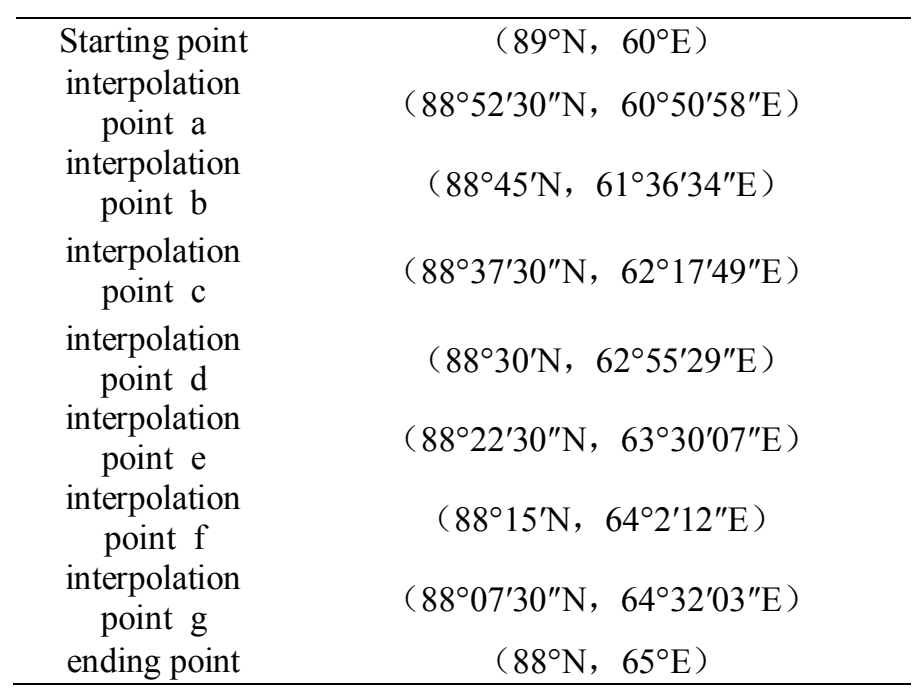


The results of the experiment show that the rhumb line can be plotted by using the method of "drawing curves by straight lines" on Equidistance Cylindrical Projection plane. In addition, because of easy operation and calculation, this method can be used to plot the rhumb line on the paper chart.

\section{Azimuth and distance measurement on the chart}

In the process of ship navigation, it's necessary for ship positioned and planning routes to measure the azimuth and distance between two points. But angle and length both exist deformation on Equidistance Cylindrical Projection plane and these disadvantages cause great difficulty to azimuth and distance measurement on the chart. So the paper puts forward a method of measuring azimuth and distance combined with the method of rhumb line plotted on Equidistance Cylindrical Projection chart.

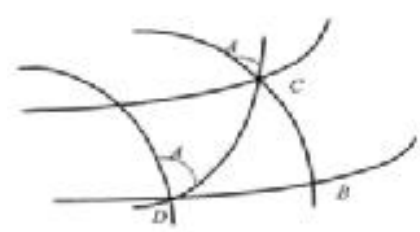

(a)

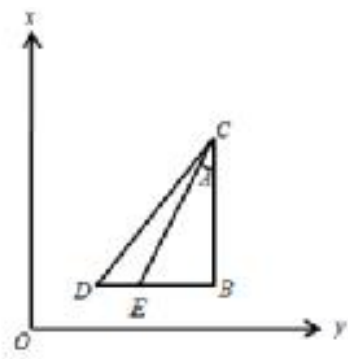

(b)

Figu.5 The sketch of rhumb line differential triangle on ellipsoid and projection plane

As is shown in Fig.5, (a) is the sketch of rhumb line differential triangle on ellipsoid and (b) is the sketch of rhumb line differential triangle on projection plane. $D C$ is a rhumb line and its azimuth is $A$, it is known the formula (6) from (a).

$\tan A=\frac{S_{D B}}{S_{B C}}$

In the formula, $S_{B C}$ represents the differential arc length of meridian and $S_{D B}$ represents the differential arc length of parallel.

In (b), the distance of $B C$ is $\Delta x$, the distance of $D B$ is $\Delta y$. There is no length deformation on the warp direction, there is length deformation on the parallel direction and its length ratio is $n$, so the distance of $B E$ is $\Delta y / n$. Thus it shows the formula (7).

$$
\left\{\begin{array}{c}
S_{D B}=\Delta y / n \\
S_{B C}=\Delta x
\end{array}\right.
$$

Substituting Formula(7) in formula (6), the formula (8) can be obtained.

$\tan A=\frac{\Delta y / n}{\Delta x}$

Spherical distance and azimuth can be seen as distance and azimuth on the plane within 30 kilometers [8]. Therefore, when the field distance of rhumb line (drawing curves by straight lines) is less than 30 kilometers, just measuring the distance of $D B$, then calculating the length ratio $n$ of the $D B$ latitude, and finding the point $E$, connecting $C E$, angle $A$ is the azimuth of this rhumb line. The distance of the rhumb line is the meridian arc length between two points multiplied by the secant of the azimuth[5], so the distance of $C E$ is the distance of the rhumb line $D C$.

About the length ratio $n$, it can be obtained from the formula(9).

$n=\frac{r_{0}}{r}=\frac{\cos B_{0} \sqrt{1-e^{2} \sin ^{2} B}}{\cos B \sqrt{1-e^{2} \sin ^{2} B_{0}}}$

Because of the small amounts of $e^{2}, \sqrt{1-e^{2} \sin ^{2} B}$ basically equal between latitude $75^{\circ}$ to latitude $90^{\circ}$, so the formula(9) can be simplified to the formula(10). 


$$
n \approx \frac{\cos B_{0}}{\cos B}
$$

This method is applied to the rhumb line in Fig.3, the azimuth and distance of this rhumb line can be directly obtained. The results are in the Table 5.

Table 5. The calculation results (1)

\begin{tabular}{ccc}
\hline & Azimuth & Distance $(\mathrm{m})$ \\
\cline { 2 - 3 } The real value & $172^{\circ} 49^{\prime} 30^{\prime \prime}$ & $112584 \mathrm{~m}$ \\
The calculated & $170^{\circ} 06^{\prime} 09^{\prime \prime}$ & $113381 \mathrm{~m}$ \\
value & $2^{\circ} 43^{\prime} 21^{\prime \prime}$ & $797 \mathrm{~m}$ \\
Error value &
\end{tabular}

Because the distance of this rhumb line is larger than 30 kilometers, the result error is larger.

Combined with the method of drawing curves by straight lines, inserting several points in the rhumb line by using Table 4,calculating the azimuth and distance in each piece respectively, the results show in the Table 6.

Table 6. The calculation results (2)

\begin{tabular}{ccc}
\hline & Azimuth & Distance $(\mathrm{m})$ \\
\cline { 2 - 3 } 1 & $172^{\circ} 24^{\prime} 00^{\prime \prime}$ & $14085 \mathrm{~m}$ \\
2 & $172^{\circ} 26^{\prime} 44^{\prime \prime}$ & $14084 \mathrm{~m}$ \\
3 & $172^{\circ} 28^{\prime} 57^{\prime \prime}$ & $14083 \mathrm{~m}$ \\
4 & $172^{\circ} 30^{\prime} 46^{\prime \prime}$ & $14082 \mathrm{~m}$ \\
6 & $172^{\circ} 32^{\prime} 17^{\prime \prime}$ & $14081 \mathrm{~m}$ \\
7 & $172^{\circ} 33^{\prime} 35^{\prime \prime}$ & $14080 \mathrm{~m}$ \\
8 & $172^{\circ} 34^{\prime} 32^{\prime \prime}$ & $14080 \mathrm{~m}$ \\
& $172^{\circ} 35^{\prime} 40^{\prime \prime}$ & $14079 \mathrm{~m}$ \\
\hline The real & Average: $^{\prime}$ & Summation: \\
value & $172^{\circ} 30^{\prime} 49^{\prime \prime}$ & $112654 \mathrm{~m}$ \\
\hline Error & $0^{\circ} 19^{\prime} 30^{\prime \prime}$ & $112584 \mathrm{~m}$ \\
value & & $70 \mathrm{~m}$
\end{tabular}

Adopting the method of calculating the azimuth and distance in each piece, the error of the result is smaller and the result can meet the requirements for ship navigation that azimuth error is less than $0.5^{\circ}$ and distance error is less than $0.2 \mathrm{~nm}$ [9]. In addition, because of easy operation and calculation, this method can be used to measure azimuth and distance on the paper chart.

\section{Summary}

Aiming at the problem that the current polar projections is not completely suitable for polar nautical chart plotting and using, the paper analyses and summarizes it. Then combining with the existing method of chart plotting and using, the paper puts forward to choose Equidistance Cylindrical Projection as the projection of Polar Chart, discusses and researches the applicability of this projection and the shortage of the application aspects. The shortage is mainly rhumb line plotted and azimuth, distance measurement, the paper analyses them and puts forward the corresponding solutions. At the same time, some specific examples prove the feasibility of the corresponding solutions. At last, the paper lays foundations for this projection application in polar chart. But the method which the paper puts forward is more complex than the current using method of the chart, so the next step will be to simplify and rule the method which the paper puts forward under the condition of meeting the marine requirements. 


\section{References}

[1] Li Shujun\&Zhang Zhe\& Li Huiwen\& Wu Di\& Wang Wei.2012. Research on Compilation of Nautical Charts of Arctic Regions, Hydrographic Surveying And Charting,32(1): 58 60.

[2] P.C. Beresford.1953. Map Projection Used in Polar Regions, Journal of Navigation, 6(1): $29 \sim 37$.

[3] Athanasios Professional.2012. Map Projections for Electronic Navigation and Other Marine GIS Applications. International Hydrographic Review,8(2): 7 19.

[4] Wen Chaojiang\& Bian Hongwei\& Chen Zhihong\& Qian duo.2014. Study on Availability of Mercator Projection in Polar Nautical Charts' Compilation, Hydrographic Surveying And Charting, 34(3): 56 59.

[5] Li Guozao\& Yang Qihe\& Hu Dingquan.1993. Map Projections, PLA Press, Beijing, China.

[6] Li Houpu.2010. The Research on the Precise Calculation Theory of Geodetic Coordinate System and Its Application Based on Computer Algebra System, Naval University, Wuhan, China.

[7] Li Jingjie.1999. GB12320-1998. Specifications for Chinese Nautical Charts, Publishing House of Chinese Standard, Beijing, China.

[8] Hua Tang.1985.Mathematical Basis for Chart, Compass Department of the Admiralty, Tianjin, China.

[9] Tian Zhengpu.2001. Basic Knowledge of Navigation, Dalian Maritime University Press, Dalian, China. 